\title{
The Planetary Vaporization Event Hypothesis: Supercharging Earth's Geothermal Core, Identifying Side Effects Blast Patterns, and Inferring how to Find Earth-Like Planets or Identifying Super Charged Geothermal Cores and their Byproduct Blast Patterns
}

\author{
Aaron R. Hurst. \\ 505 S. Everina Cir, Brandon, FI 33510. \\ ARHurst2021@gmail.com \\ ORCID 0000-0001- 8216-7696
}

\begin{abstract}
Keywords: Calcium-aluminum-rich inclusions, Chondrules, Earth, Earth-like, Earth Mark One, Earth Mark Two, Epsilon-Eridani, Eta-Corvi, Interplanetary Dust Cloud, Late Heavy Bombardment, Main Belt, Moon, Outer Belt, Primary Subduction Zone, Secondary Subduction Zone, Tungsten Isotope, Wayward Moon
\end{abstract}

\begin{abstract}
The supercharged nature of the Earth's geothermal core can be demonstrated by three thought experiments exhibiting it is tremendously more powerful than any other terrestrial object in the solar system (planet or moon). Identifying a minimum of four byproduct asteroid blast patterns linked to the formation of Earth's supercharged geothermal core is critical to properly identifying stars that also have these four byproduct asteroid blast patterns. These stars are the most likely to host an Earth-like planet qualified by having a supercharged geothermal core.
\end{abstract}

The Planetary Vaporization-Event (PVE) Hypothesis provides a basis for correlation between the supercharged nature of Earth's geothermal core and at least 14 listed side effects: (1) the asteroidwide/planet-scale homogenization and lack thereof of ${ }^{182} \mathrm{~W} \varepsilon$ for Earth, the Moon, Mars and meteors, (2) the primary and secondary shifting of Earth's tectonic plates, (3) the solar system wide displacement of Earth's wayward moons (including Ceres, Pluto, Charon and Orcus) outgassing identical samples of ammoniated phyllosilicates, (4) the formation of asteroids at $100+$ times the expected density of a nebular cloud vs. pre-solar grains formation density at the expected density of a nebular cloud, (5) three distinct formation timestamps for all known asteroids within a 5 million year window $4.55+$ billion years ago, (6) the estimated formation temperature of CAI at 0.86 billion Kelvin and (7) the remaining chondritic meteorite matrix flash vaporizing at 1,200-1,900 ${ }^{\circ} \mathrm{C}$, (8) followed by rapid freezing near $0 \mathrm{~K}$, (9) the development of exactly 2 asteroid belts and a swarm of non-moon satellites, (10) particulate size distinction between the 2 asteroid belts of small/inner, large/outer, (11) the proximity of the Trojan Asteroid Groups to the Main Asteroid Belt, (12) observation of a past or present LHB, (13) the development of annual meteor showers for Earth proximal to apogee and/or perigee, (14) the Sun being the most-likely object struck by an asteroid in the inner solar system.

Through better understanding of the relevant data at hand and reclassification of the byproducts of supercharging the core of a planet, at least 5 new insights can be inferred and are listed as: (1) the original mass, (2) distance and (3) speed of Earth Mark One, (4) the original order of Earth's multi-moon formation and (5) the high probability of finding detectable signs of life on a planet orbiting the stars Epsilon Eridani and Eta Corvi.

There are at least 6 popular hypothesis that the PVE Hypothesis is in conflict with, listed they are: (1) a giant impact forming the Moon, (2) asteroids being the building blocks of the solar system, (3) the Main Asteroid Belt being the result of a planet that never formed, (4) the LHB being a part of the accretion disk process, (5) the heat in Earth's core coming primarily from the decay of radioactive elements, (6) the Oort Cloud being the source of ice comets. 


\section{Introduction}

Earth is extraordinary for its ability to host a wide variety of life forms and habitats. Its supercharged geothermal core, serviceable gravity, water, and hydrothermal vents make Earth a suitable refuge for evolving life forms for potentially tens of billions of years. Necessary for life on Earth, the dynamic geothermal core provides a heated environment even in places the Sun's energy cannot reach allowing the conversion of thermal energy to food energy, and is a key component in creating a magnetosphere produced by Earth's dynamo system (Hess, 1968), which in turn provides protection from the Sun's radiation. Thus, Earth's geothermal core provides three necessities for life: warmth, food, and protection. In this solar system, only Earth has these key ingredients for a lifesustaining environment.

The positive side-effects of Earth's core for life are fundamental however, many are neutral or even harmful to life. Because many of the side-effects are misidentified, it is necessary to examine each one and how they were the results of the process of creating Earth's supercharged geothermal core. This will help to better understand and classify objects in the solar system.

Of the many side-effects when supercharging the core of a planet, volcanoes are a necessary danger. Io, the inner most moon of Jupiter, comes the closest to Earth's count of active volcanic hotspots, with 170 known active hotspots (Williams et al., 2011). In comparison, Earth has 1,500 known active volcanoes, of which 500 are erupting at any given time, many underwater (Schmidt, 2004). Further demonstrations of the supercharged nature of Earth's geothermal core include its longevity, its relative volume of resurfacing, and its energy output.

Evidence provided and examined for a new scientific hypothesis relative to Earth's reformation: the Planetary Vaporization Event (PVE) Hypothesis, includes but is not limited to:

(1) asteroid-wide/planet-scale homogenization of ${ }^{182} \mathrm{~W} \varepsilon$ isotopic data (Halliday, 2000; Kruijer et al., 2015; Windley, 1970; Youngl et al., 2016), (2) identifying primary and secondary subduction zones as well as divergent boundaries (Domeier and Torsvik, 2014), (3) the identical out-gassing from three dwarf planets (Sanctis et al., 2015), (4) asteroid formation at 100+ times the density of expected nebular cloud (Alexander et al., 2008), (5) all known asteroids, comets and meteorites having three distinct timestamps of $4.55+$ billion years within a 5 million year window (Amelin and Krot, 2007), (6) calcium aluminum-rich inclusion found only within the oldest of chondrite meteors showing effects of neutron-burst nucleosynthesis in a massive star at a high peak temperature of $0.86 \times 10^{9} \mathrm{~K}$ (Quitté et al., 2007), (7) the remaining chondritic meteorite matrix was exposed to flash melting temperatures ranging from 1,600 to $1,800{ }^{\circ} \mathrm{C}$ (Hewins and Radomsky, 1990), (8) meteoriteexclusive minerals experienced rapid cooling at near $0 \mathrm{~K}$ (Anders, 1964), (9) observations of two asteroid belts and gas planet satellites, (10) with distinctly different particle sizes suggesting the inner belt as the source of mass (Duchene et all., 2014) visually producing zodiac lights and gegenschein along the plane of the inner solar system, (11) the approximation of the Trojan Asteroid groups to Earth's hypothesized original orbital location, (12) evidence of a current or past LHB (Gomes et al., 2005; Lisse et al., 2012), (13) and a 3D solar system simulator ${ }^{1}$ confirming annual meteor showers occur after an orbital adjustment, (14) as well as finding the Sun to be the most likely object struck by asteroids, consistent with the findings of Gomes et al., 2005 (this is useful when inferring the mass of Earth Mark One).

\footnotetext{
${ }^{1}$ A planet-vaporizing event requires more energy than can be reproduced in a modern laboratory. A simulation also creates an easily reproduced variable experiment capable of presenting random outcomes for unbiased observations. To access 3D Solar System Simulator 1.3, go to https://drive.google.com/open?id=0BwnpKNsUN4EJT0Z1M0p2dlZPbEU. Note: Replays of recordings longer than 70 years are not possible with this program.
} 
New information that can be deduced by viewing the aforementioned data as being a byproduct of supercharging the core of Earth includes but, is not limited to: (1) The original mass, (2) distance and (3) speed of Earth Mark One, (4) the original order of Earth's wayward moons and likely the greatest use for all of this knowledge, (5) the high probability of detecting carbon, hydrogen, oxygen and nitrogen (CHON/life) on a planet orbiting Epsilon Eridani and Eta Corvi.

\section{Demonstrations of the Supercharged Nature of Earth's Geothermal Core}

Three thought experiments demonstrate beyond any reasonable doubt the power of Earth's geothermal core and its subsequent qualification as "supercharged." These concern (1) its longevity, (2) a comparison of the volume and size of Olympus Mons vs Hypothetical Hawaii Supreme, and (3) a comparison of the energy output over time of the Earth vs the Sun.

(1) In terms of longevity, Verhoogen (1980) stated the average core $(6,000 \mathrm{~K})$ and mantle $(4,000 \mathrm{~K})$ temperatures have cooled $250 \mathrm{~K}$ since the formation of the Solar System approximately 4.55 billion years ago. Isolated from external sources and at a cooling rate of $55 \mathrm{~K}$ per billion years, the Earth's average core temperature will take approximately 86 billion years to cool to $0 \mathrm{~K}$. The Sun is estimated to nova in approximately 7.6 billion years. The length of one Sun cycle can be estimated to be approximately 12.15 billion years. Thus, Earth's geothermal core has the potential to last longer than seven Sun cycles or approximately 90.9 billion years.

(2) An examination of Olympus Mons (Mars) and Maat Mons (Venus) reveals their sizes are due to the absence of moving tectonic plates on their respective planets. Although this requirement, among others like not sinking into the mantle under its own weight (isostatic adjustment) or eroding, needed to produce the hypothetical Hawaii Supreme can never be met on Earth, a comparison of it to Olympus Mons demonstrates the supercharged nature of Earth's geothermal core. Olympus Mons is the largest known shield volcano in the solar system, with an approximate volume of $1.7 \times 10^{6} \mathrm{~km}^{3}$ (Isherwood et al., 2012). During the last 85 million years, the Hawaiian hotspot has produced an estimated yield of over $750,000 \mathrm{~km}^{3}$ of lava in the volcanic Hawaiian Emperor seamount chain (Clague and Dalrymple, 1987). If the Hawaiian hotspot began 50,000 million years after Earth's reformation events and continually deposited at the same location and rate over a course of 4.5 billion years, the volume of the resulting shield volcano would be approximately $4.0 \times 10^{10} \mathrm{~km}^{3}$ or 23 times greater than that of Olympus Mons. The height of Olympus Mons is nearly $25 \mathrm{~km}$. Multiplying $25 \mathrm{~km}$ by the cubed root of 23 is used as the base measurement to estimate the height of Hawaii Supreme to be $71.09 \mathrm{~km}$ at its summit.

(3) According to Davies and Davies (2010), Earth's core contains enough energy to release $47 \pm 2$ TW to this day. Given the Earth's age of 4.55 billion years and a consistent power output of $47 \pm 2 \mathrm{TW}$, the Earth has produced energy at an average rate of $6.7439736 \times 10^{18} \mathrm{TW}$ over the course of its existence. Ignasi's (2010) estimate of the Sun's energy output was approximately $3.86 \times 10^{14} \mathrm{TW}$. Thus, in the course of its existence, Earth has produced as much energy as the Sun produces in approximately 4.85 hours. However, this underestimates Earth's actual release of energy. In the past, Earth has expelled energy at a rate of more than $47 \pm 2 \mathrm{TW}$, likely due to the loss of its primordial heat through the escape of approximately $20 \%$ of its geoneutrinos into space (Dye, 2012). Thus, although based on different time scales, the methodology applied here suggests it takes 260,159 years for Earth to produce as much energy as the Sun produces in one second.

\section{Planetary Vaporization Event Hypothesis vs. Other Unsolved Hypothesis}

The data collected exploring the PVE hypothesis supports an overlooked connection of cause and effect existing between the super charged core of Earth and many aspects of the Solar System thought to be unrelated to each other. While the cause of supercharging Earth's core is yet to be determined, many of its effects can be more accurately labeled. Among the most revealing effects are the physical degree of various celestial object's homogenization of ${ }^{182} \mathrm{~W} \varepsilon$ isotopes (Halliday, 2000; Kruijer et al., 2015; Windley, 1970; Youngl et al., 2016), Earth's high level of tectonic activity 
(Wegener, 1912), Earth's existence as the only terrestrial planet with a large moon (Canup and Asphaug, 2001; MacKenzie, 2003), the late heavy bombardment (Gomes et al., 2005), and the Main (Von Humboldt, 1850) and Outer (Fernandez, 1980) asteroid belts.

The PVE hypothesis is consistent with all available data leaving open the plausibility that the chaos of the LHB, the distinctly different particulate size patterns of the asteroid belts, the annual occurrence of meteor showers, and the supercharged nature of Earth's geothermal core are all the result of one series of planet-reforming events that impacted Earth Mark One approximately 4.55+ billion years ago. These events resulted in the development of a relatively smaller planet with shifting tectonic plates and one enduring large moon. The series of cataclysmic events resulted in releasing the rest of the moons of Earth Mark One to develop new independent orbits around the Sun, becoming wayward moons (currently labeled as dwarf planets).

To better understand the plausibility of the PVE hypothesis, it is helpful to examine the preexisting hypotheses for the origins of the previously listed celestial objects including (1) Earth's large Moon, (2) asteroids themselves, (3) the two asteroid belts, (4) the LHB, (5) the source of heat for Earth's supercharged geothermal core, and (6) the Oort Cloud as the source of ice comets, all of which have remained unsolved. None of the existing hypotheses can explain the formation of all these anomalies from a single explanation. In addition, several of the explanations are different enough that if one of the hypotheses is correct, the other hypotheses cannot be correct. For example, there are five debated hypotheses for the origin of the Moon which cannot correlate (capture, co-formation, colliding planetesimals, fission and giant impact).

(1) Of the five most prevalent natural hypotheses for the formation of Earth's moon, many scientists favor the giant impact hypothesis, which states that the Moon was created when Earth was struck by Theia, a Mars-sized planet (Canup and Asphaug, 2001, MacKenzie, 2003), just over 4.5 billion years ago. Although both the PVE hypothesis and the giant impact hypothesis suggest Earth was struck by a tremendous force, the two hypotheses differ concerning whether the Moon predates the event or is a product of it.

(2) Hypothesis of how the majority, approximately $86 \%$ of fall meteorites (observed falling) came to contain chondrites while a portion of the remaining $14 \%$ shows signs of extreme formation patterns like shockwaves are considered unsatisfactory (Karner and Watts. 1983; MacPherson et al., 2005; Krot et al., 2009). This is likely resulting from the contending view of asteroids as being the building blocks of the solar system and the Sun as the source of the extreme heat exposure to which they were subjected. The deficiencies in the current understanding include the inability to explain how all asteroids reconstituted near the Sun's corona before changing their orbits to develop into planets, asteroids belts, and retrograde comets. Instead, the findings are consistent with the source of the high-density, vaporization temperatures recorded in meteorites as coming from the exploding Earth Mark One. Records from meteorite samples show the formation of all known asteroids occurred from three distinct formation events (Amelin and Krot, 2007) within a five-million-year window of development that occurred from 4568.22 \pm 0.6 to $4564.7 \pm 0.6$ million years ago (Amelin et al., 2002; Bouvier and Wadhwa, 2010; Connelly et al., 2012; Schrader and Desch, 2016). Asteroids are too young to be the building blocks of planets. The discovery of pre-solar grains (Magill, 1990) provide an age and material appropriate substance that better fits what could be expected from a planetary building block.

(3) Heinrich Olbers's discovery in 1802 of Pallas in the Main asteroid belt and his subsequent work suggest the less popular hypothesis that the Main Belt is the remains of a destroyed planet (Herschel, 1807) rather than the more popular hypothesis (Phaeton) that it is a planet never finished due to the influence of Jupiter's gravity (Kirkwood, 1867). Identifying Earth as the planet located and destroyed at the Main Belt is consistent with the less popular view.

(4) According to another theory based on scientific exploration, asteroids are the leftover unused building blocks of the planets and solar system (National Research Council, 2011). Analysis of meteorite data shows asteroids are among the oldest known objects in the Solar System. Over 
4.55 billion years ago, they were exposed to extraordinarily hot conditions (hot enough to sublimate tungsten and aluminum at 1,200-1,900 ${ }^{\circ} \mathrm{C}$ [Hewins and Radomsky, 1990]) and then just as suddenly cooled inexplicably quickly (Anders, 1964). However, how this rapid cooling took place was unclear to Anders in 1964. In 2005 the work of Gomez et al, suggested that after a 700-million-year quiescent period the rapid migration of the outer giant gas planets could have triggered the LHB. The PVE Hypothesis proposed solution to the source of mass for the LHB is that the vaporization and sudden ejection of such a large volume of Earth Mark One's mass into the cold of space caused the cloud of cooling vapor to condense or immediately solidify into small enough pieces that rapidly froze before reforming into larger asteroids or breaking down into smaller asteroids.

(5) One of the favored explanations for the source of fuel that keeps Earth's geothermal core going is the decay of radioactive elements such as uranium, thorium and potassium. However, in 2011, The KamLAND Collaboration published results concluding only half of Earth's energy output can be accounted for by radioactive decay (Gando et al., 2011), sighting the rest as coming from an unknown source or the result of primordial heat from the formation of the planet.

(6) The shedding of Earth Mark One's mass to become Earth Mark Two provides an alternative solution to the Oort Cloud hypothesis (Dones et al., 2004) as the source of long-period ice-comets orbiting the Solar System in a retrograde ellipsis. Furthermore, this identifies the accretion disk as the source of water Earth Mark One received and then later ejected as part of the LHB, asteroid belts, and long-period comets. Water falling from space to Earth Mark Two is water that once was already a part of Earth Mark One. Moreover, the Oort cloud has not been observed in this solar system or any other solar system, not even in one solar system that an Oort cloud might have been expected to be more observable in, like Epsilon Eridani (Potemine, 2010).

A massive Earth Mark One with multiple moons experiencing a planet-scale vaporization event resulting in the transformation to a smaller Earth Mark Two is a single hypothesis for the source of primordial heat in Earth's core, the releasing of all except the closest (and most dense) of Earth's moons, the massive amount of ejected planetary debris that developed into the LHB, two asteroid belts, the Jupiter Trojan Asteroid Groups, the smaller dwarf planets (like Vista), captured satellites orbiting planets (e.g., Phobos and Deimos of Mars, Himalia and Amalthea of Jupiter, Phoebe and Hyperion of Saturn), and retrograde objects (e.g., comets, asteroids, annual meteor showers).

\section{Analysis of Existing Data}

The complete list of 14 direct blast pattern side effects caused by supercharging Earth's core included in this manuscript are; (1) The asteroid-wide/planet-scale relative homogenization of ${ }^{182} \mathrm{~W}$ $\varepsilon$ isotope samples from Earth and all known asteroids vs the less homogeneous ${ }^{182} \mathrm{~W} \varepsilon$ isotope samples from the Moon and Mars, (2) a primary subduction zone developing a primary divergent boundary, manifesting a secondary subduction zone which in turn creates a secondary divergent boundary (3) the outgassing of identical ammoniated phyllosilicates from three suspect wayward Earth moons, Orcus, Charon, and Ceres, (4) asteroids formed at 100+ times the expected density of a nebular cloud, (5) three distinct timestamps for asteroid formation within a 5 million year window, (6) calciumaluminum-rich inclusion (CAI) suspected formation temperature of 0.86 billion $\mathrm{K}$ vs. the near $0 \mathrm{~K}$ formation temperatures of pre-solar grains which did form at the expected density of a nebular cloud or accretion disk, (7) the remaining chondritic meteorite matrix samples tested flash melting from 1,400 to $1,750{ }^{\circ} \mathrm{C},(8)$ rapid freezing near $0 \mathrm{~K}$, (9) the formation of two asteroid belts and gas planet satellites, (10) with distinctly different particle sizes producing zodiac lights and gegenschein along the plane of the inner solar system visible to the naked human eye, (11) approximation of the Trojan Asteroid Groups to Earth Mark Ones destruction (12) evidence of a past LHB, (13) confirmed by a virtual 3D simulation create to investigate the PVE hypothesis, annual meteor showers resulting after a planet changes its orbit as well as (14) simulating the Sun to be the object most likely struck by a LHB. 


\subsection{Tungsten Isotropic Data}

182 tungsten isotropic data supporting the creation of the Earth's moon prior to the asteroidwide/planet-scale melting event that resulted in homogenization of the Earth has been collected from meteorites, and rock samples from Earth, the Moon, and Mars.

Samples of Martian meteorites $(-1.0$ to $+3.25 \varepsilon)$ and lunar rocks $(-2.25$ to $+7.0 \varepsilon)$ exhibit a broader range of ${ }^{182} \mathrm{HF}-{ }^{182} \mathrm{~W} \varepsilon$ values than samples from ancient Earth $(1.0$ to $+3.0 \varepsilon)$, modern Earth $(-2.0$ to $+1.75 \varepsilon)$, and fall meteorites $(-4.0$ to $+2.0 \varepsilon)$ (Halliday, 2000; Kruijer et al., 2015; Windley, 1970; Youngl et al., 2016) as shown in Figure 1. The available data is consistent with all planets and moons forming from the accretion disk having a greater range of ${ }^{182} \mathrm{~W} \varepsilon$ values. Earth's shift from positive ${ }^{182} \mathrm{~W} \varepsilon$ to negative ${ }^{182} \mathrm{~W} \varepsilon$ over time is consistent with the PVE Hypothesis and make evident neither Mars nor Earth's Moon experienced the vaporization/homogenization process that affected Earth. Earth having a tight grouping of ${ }^{182} \mathrm{~W} \varepsilon$ values (both in ancient and modern times) and the Moon not having such a tight grouping is consistent with Earth being struck by a planet-scale melting event that did not result in the creation of the Moon. The Moon predating this planet-reshaping event is consistent with Earth Mark One being large enough to produce more than one moon in the natural way large planets form more than one moon during the accretion process before the vaporization/homogenization event greatly reduced Earth's size. Earth's shift from a greater value of ${ }^{182} \mathrm{~W} \varepsilon$ to a lesser value of ${ }^{182} \mathrm{~W} \varepsilon$ over time also suggest asteroid and meteorite bombardments are the sources of contamination in both the planet and Moon samples and are not the source of planetary building material.

Earth has had two different clusters of ${ }^{182} \mathrm{~W} \varepsilon$ values separated by billions of years. These clusters are attributable to the ongoing planet-scale homogenization of Earth and the lack of dynamic geothermal cores in the Moon and Mars, which affected Earth's degree of mixing of ${ }^{182} \mathrm{~W} \varepsilon$ contamination into the mantle for over four billion years (Halliday, 2000; Kruijer et al., 2015; Windley, 1970; Youngl et al., 2016). Data associated with the eventual development of Earth's supercontinent into Pangaea (Weijermars, 1989) and its predecessors show Earth was chemically contaminated by continual chondritic bombardment. Although Precambrian samples are less contaminated and therefore have a greater amount of ${ }^{182} \mathrm{~W} \varepsilon$, the same data demonstrates planet-scale homogenization of Earth was already underway over four billion years ago. The lack of even a single Earth sample predating the homogenization event suggests all the remaining solid portions of Earth Mark Two experienced post-event melting and resurfacing. 


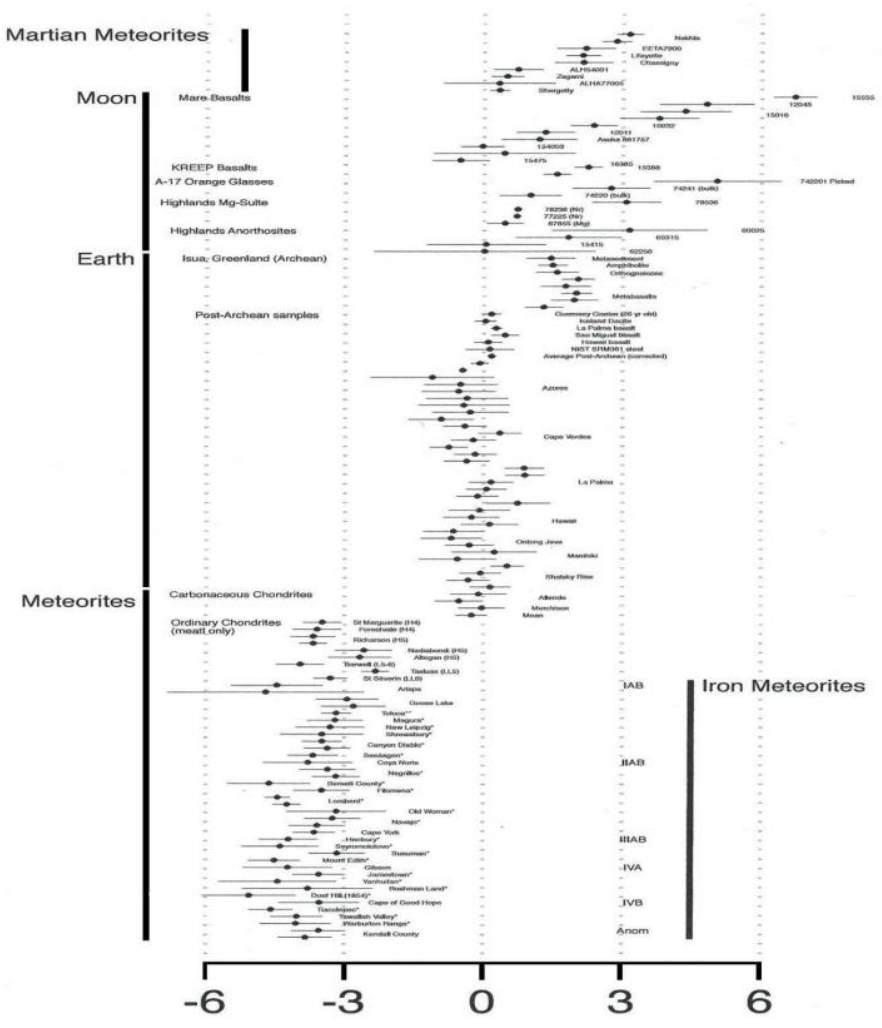

Figure 1. Ratios of ${ }^{182} \mathrm{~W} \varepsilon$ to ${ }^{184} \mathrm{~W} \varepsilon\left({ }^{182} \mathrm{~W} \varepsilon\right)$ in meteorites, modern Earth, ancient Earth, the Moon, and Mars (Halliday, 2000; Kruijer et al., 2015; Windley, 1970; Youngl et al., 2016)

\subsection{The Moving Surface of Earth}

An effect of Earth's supercharged geothermal core observed only on Earth is its ability to shift very large tectonic plates on the surface against each other. Today's most active plate that has the most energy-in-motion from the Earth vaporization event and then the following two orbit changing impacts 4.55 billion years ago is the Pacific Plate. The Pacific plate has more volcanoes, hotspots, earthquakes, and tsunamis around its rim than around any other tectonic plate on Earth.

Today, the most powerful zone is a subduction zone that stretches along the east coast of New Zealand, around Papua to Malaysia, then north to China, across the east of Japan and the Kamchatka Peninsula and finally ends at the Gulf of Alaska. The subducting plate moves at an average rate of approximately $8.5 \mathrm{~cm}$ per year. In the Paleozoic Era, the supercontinent Gondwana experienced subduction moving south along its eastern coast (the Australian Plate) and along the west coast of Laurussia (North America; Domeier and Torsvik, 2014). The long- standing history of the Pacific Ring of Fire and its greater speed relative to any other subduction or divergent zones qualify it as Earth's primary subduction zone.

The fastest moving divergent boundary on Earth is the East Pacific Rise, which moves at a rate of approximately $6 \mathrm{~cm}$ a year. It is hypothesized that the primary divergent boundary is located in opposition to the primary subduction zone (New Zealand to Alaska), because it is a response to it. In turn, this primary divergent boundary creates a secondary subduction zone at the Peru-Chile Trench. The Indian Plate, which is experiencing a secondary divergent boundary, moves northeast at approximately $5 \mathrm{~cm}$ a year. As a result, the Indian and Australian Plates have created a second secondary subduction zone under the Eurasian and Pacific Plates.

\subsection{Outgassing of Earth's Lost Moons}

The known dwarf planets include Pluto, Charon, Makemake, Ixion, Eris, Orcus, Quaoar, Sedna, 2002TC302, 2002Ux25, and Ceres. However, wayward moon may be a better term for some of these 
dwarf planets. Excluded from the possibility of being wayward moons are any dwarf planets ovoid in shape and spinning end over end. These planets were obviously formed by rapidly cooling from a liquid state to a solid while under the stress of their own centrifugal force (Haumea). Also excluded from being wayward moons are any dwarf planets containing CAI and chondrites because they were inexplicably formed from vaporizing temperatures.

Recent enhanced analysis suggests the Moon and Earth began with similar W and $\mathrm{O}$ isotopic compositions. Earth's composition changed significantly after it was struck and exposed to chondrite bombardment (Touboul et al., 2015; Weizsäcker, 1948; Windley, 1970). Lunar petrology records indicate the Moon, as well as the inner planets, experienced a sharp spike in cataclysmic bombardment 700 million years after its formation (Gomes et al., 2005).

Of all the dwarf planets, Ceres is unique in its location at the hypothetical location of the original orbit of Earth Mark One. Based on its size, density, and composition, it is not a large asteroid. However, without rock samples to compare its $\mathrm{O}$ and W isotopic and HSE compositions with those of Earth and the Moon, other effective ways to investigate the possibility of Ceres being a wayward moon of Earth are (a) to detect Earth's Moon outgassing the identical ammoniated phyllosilicates Orcus, Charon, and Ceres have and (b) collect rock samples from Ceres.

Evidence supporting the Moon predates the Earth vaporization event is twofold. First, the Moon's ${ }^{182} \mathrm{~W} \varepsilon$ isotope homogenization is disproportionate to the level of homogenization Earth was exposed to. Second, the oldest Moon rocks are older than any Earth rock sampled and are relatively close in age to asteroids as well as the average estimated age the Sun achieved fusion (approximately 90 million years younger). This implies asteroids are not old enough to have been the pre-solar material needed to create the Sun, Moon, planets and the rest of the solar system during the accretion disk process.

The most compelling physical evidence that Ceres's origin is connected to the origins of other dwarf planets is the outgassing of ammoniated phyllosilicates detected on Orcus, Charon, and Ceres (Sanctis et al., 2015). The similarities in chemical outgassing suggest the dwarf planets must have formed near each other. Sanctis et al. (2015) further speculated Ceres originally developed as a transneptunian object (TNO) and was later deposited at the Main Belt. The ammoniated phyllosilicates do not appear to have come from the asteroid belt by means of meteoric bombardment. Pluto's moon Charon and the anti-Pluto Orcus have ammoniated phyllosilicates in abundance which is to be expected when TNOs hold onto their ammoniated phyllosilicates more easily than Ceres, which is closer to the Sun at the Main Belt. Although dwarf planets sharing similar chemical compositions is consistent with the hypothesis of all dwarf planets being formed near one another as moons of the same planet, the PVE hypothesis suggests another opposite view. Pluto, Charon, Makemake, Ixion, Eris, Orcus, Quaoar, Sedna, and possibly more dwarf planets developed near the Main Belt, along with Ceres, as moons of Earth Mark One and all except Ceres, Earth and the Moon were later relocated to trans- Neptunian orbits. In a measure of agreement, both opposing hypotheses suggest dwarf planets originally formed in close proximity to each other. Only one hypothesis goes further to indicate Earth Mark One as the planet and source of gravity associated with their formation.

One outgassing experiment that has the potential verify the PVE hypothesis would be to dig into the Moon's surface (preferable in locations where the Sun never shines) until out- gassing can be detected and spectral analysis comparisons can be made against the out-gassing of ammoniated phyllosilicates from Ceres, Orcus and Charon. A resulting match from such an experiment will serve to determine whether the dwarf planets are indeed Earth's wayward moons. A different way to verify the premises of the PVE hypothesis will require the construction of spacecraft capable of collecting rock samples from Ceres and returning them to Earth for analysis or landing a laboratory on Ceres capable of conducting comparative experiments analyzing samples for similarities in HSE, W and O isotopes with samples from Earth and the Moon. 


\subsection{Asteroid Formation Density vs. Nebular Cloud}

The abstract for "The Formation Conditions of Chondrules and Chondrites" (C. M. O'D. Alexander, J. N. Grossman, D. S. Ebel, F. J. Ciesla) provides a simple and effective description explaining what their expectations were vs the results of their testing of chondritic meteorites.

"Chondrules, which are roughly millimeter-sized silicate-rich spherules, dominate the most primitive meteorites, the chondrites. They formed as molten droplets and, judging from their abundances in chondrites, are the products of one of the most energetic processes that operated in the early inner solar system. The conditions and mechanism of chondrule formation remain poorly understood. Here we show that the abundance of the volatile element sodium remained relatively constant during chondrule formation. Prevention of the evaporation of sodium requires that chondrules formed in regions with much higher solid densities than predicted by known nebular concentration mechanisms. These regions would probably have been self-gravitating. Our model explains many other chemical characteristics of chondrules and also implies that chondrule and planetesimal formation were linked."

At a relatively lower density, a nebular cloud creates celestial objects like the planets by a process of cold accession. Approximately 100+ times the density, the LHB began as a 0.86 billion Kelvin exploding super-terrestrial that created meteorites by a prosses of hot accretion.

\subsection{Three Distinct Timestamps}

The chondritic composition of asteroids and comets have rarely been sampled off Earth (Stardust, Hayabusa) however, those few samples are consistent enough with findings from meteorites to suggest they share a common origin (some interesting/unique off-world samples included amino acids). Based off the work of Amelin and Krot (2007) it can be inferred " that chondrule formation started contemporaneously with or shortly after formation of CV CAIs and overlapped in time with formation of the basaltic crust and iron cores of differentiated asteroids. The entire period of chondrule formation lasted from 4566.6 $\pm 1.0 \mathrm{Ma}$ (Allende) to 4564.7 $\pm 0.6 \mathrm{Ma}$ (CR chondrite Acfer 059$)$ to $4562.7 \pm 0.5 \mathrm{Ma}(\mathrm{CB}$ chondrite Gujba) and was either continuous or consisted of at least three discrete episodes. Since chondrules in CB chondrites appear to have formed from a vapor-melt plume produced by a giant impact between planetary embryos after dust in the protoplanetary disk had largely dissipated (Krot et al. 2005), there were possibly a variety of processes in the early solar system occurring over at least 4-5 Myr that we now combine under the umbrella name of "chondrule formation."

\subsection{Hot-forged (CAI) Debris vs. Cold-forged (Pre-Solar Grains) Building Blocks}

Interpreting meteorites as Earth Mark One debris instead of unused building blocks of the accretion disk process is consistent with all available meteorite samples as a possible explanation of their composition. The conventional view is that the CAI found within chondrite meteorite samples, the oldest of all samples found within meteorites, are the building blocks of the Solar System (National Research Council, 2011). Exceptions to this view are pre-solar grains found within the Tagish Lake meteorite (Keiko et al., 2006), the Hypatia stone (Belyanin et al., 2017), and other presolar grains found within meteorites (Magill, 1990; Ott, 2002). CAI, which were formed at incredibly high temperatures of 0.86 billion K (Quitté et al., 2007) and, to a lesser degree, the chondrite formation temperature range of approximately $1,200-1,900{ }^{\circ} \mathrm{C}$ (Hewins and Radomsky, 1990), many if not most have not undergone any alteration in their composition for billions of years and are found in 85.7-86.2\% of fall meteorites (Yoneda and Grossman, 1995). This explains why researchers once thought CAI and chondrites were formed either from condensates from a solar gas (Avice et al., 2015) or from a supernova (Quitté et al., 2007).

However, the evidence against the leftover building block hypothesis includes the impact of the LHB on the surface of Earth's moon starting 700 million years after planet formation (Gomes et al., 2005) and the formation of meteorites (and presumably all asteroids and comets) in a four to five million year window during three distinct formation events (Amelin and Krot, 2007) at a density 
$100+$ times or more greater than that expected of chondrule formation within the accession disk (only 0.1 grams per cubic meter or less; Alexander et al., 2008).

The leftover building block hypothesis contains some basic flaws. The first is that CAI and chondrules are missing from even the oldest rock samples obtained from any celestial body, excluding asteroids, comets, and meteors. Lunar sample 67215 is 4.46 billion years old, which is only about 90 million years after the first asteroid formation event that resulted in the formation of CAI in chondritic meteorites. The second is that asteroids are the same age as the planets and, as such, are not old enough to have formed from the dying star that supplied the nebular cloud from which the Solar System developed. The third is that the discoveries of pre-solar grains within the Tagish Lake meteorite, the Hypatia stone, and within other meteorites are all thought to be older than the Sun (Belyanin et al., 2017; Ott, 2002) and formed under space-like cold conditions (Belyanin et al., 2017; O'Leary, 2008) at a formation density expected of a nebular cloud or accretion disk. According to Magill (1990), this provides a better example of the composition of interstellar dust older than the Sun. Not only do the isotopic compositions found therein indicate formation under conditions near absolute zero, the expected conditions that existed before the Sun achieved fusion, but the enormous range in isotopic composition also demonstrates the variety of nucleosynthesis processes in pre-solar material from which these elements were created. This composition process is quite unlike that of homogenized chondritic meteorites, which formed from a vapor cloud under extremely hot conditions followed by rapid cooling at a time long after the Sun began to from the accretion disk at a density $100+$ times greater than an accretion disk or nebular cloud.

\subsection{Flash liquidus Matrix}

"Abstract - The liquidus temperatures of chondrules range from about $1200 \mathrm{C}$ to almost $1900 \mathrm{C}$, based on the calculation of Herzberg (1979). Dynamic melting and crystallization experiments with no external seeding suggests that some chondrule textures formed with initial temperatures below the liquid (e.g. porphyric, granular) and some were completely melted (e.g. excentroradial, glassy). Type I and III chondrules in carbonaceous chondrites in this interpretation consist of incompletely melted magnesian chondrules, completely melted silica-rich chondrules and intermediate composition chondrules with both porphyritic and nonparphyritic textures. A similar pattern for ordinary chondrites, with data also for Type II porphyritic and barred olivine chondrules, suggest that few chondrules with liquidus temperatures over $1750 \mathrm{C}$ were completely melted and few with under $1400 \mathrm{C}$ were incompletely melted. The range of liquidus temperatures for barred olivine chondrules, for which initial temperatures of 1400-1750 C and, because of a peak in the distribution of barred olivine chondrule temperatures at 1500-1550 C, the temperatures appear normally distributed within this range. Given a narrow range of temperatures, bulk composition is at least as important as initial temperature in controlling chondrule textures.

Truly granular (not microporphyritic) Type I and truly glassy Type II and III chondrules appear under-represented in nature accounting to this model, based on internal nucleation experiments. External heterogeneous nucleation, or seeding due to droplet-dust collisions, is likely to occur in a dusty nebula and has been shown to reproduce chondrule textures experimentally. Generally high initial temperatures (1600-1800 C), coupled with dust-seeding of superheated droplets of less refractory composition is an alternative explanation of chondrule textures. Cooling rates of $100-1000 \mathrm{C} / \mathrm{hr}$ are required for chondrules, which must have been mass produced in clouds with sufficient particle density to buffer cooling rate and perhaps also initial temperatures. Melting precursor particles in a thick clump and/or the nebular mid-plane would provide evaporation and thus explain the high oxidation state and volatile content of chondrules, relative to the bulk hydrogen-rich nebula, as well as the nature of the cooling."

(Hewins and Radomsky, Temperature conditions for chondrule formation. 1990: Abstract) 


\subsection{Inexplicable Cooling}

"There are perhaps as many opinions as to the origin of meteorites as there are students of meteorites. Probably in no other branch of natural science is there such a wealth of observational data coupled with such a lack of unanimity in interpretation." (Wood, 1963a)

The 57 years following that quote provided advances in understanding meteorite composition that Wood could have only imagined however, no amount of new data provided the missing component needed to provide an agreeable hypothesis to the origin of meteorite formation (excluding meteorites coming from the Moon or Mars). Of the many problems in creating an agreeable model to explain the findings examined, the exposure to extreme heat and rapid cooling remained prominent.

"Cooling problem - An even more basic difficulty of the high-pressure hypothesis is the juxtaposition of high pressures and low temperatures which it requires. The gravitational pressure $\mathrm{P}$ is a celestial body of uniform density $\mathrm{p}$, at a distance from the center is $\mathrm{P}=2 / 3 \mathrm{G} \pi \mathrm{p}^{2}\left(\mathrm{a}^{2}-\mathrm{r}^{2}\right)$ where $\mathrm{a}$ is the radius of the object, and $\mathrm{G}$, the gravitational constant. If a pressure of $\geqslant 30 \mathrm{~kb}$, the lowest value permissible by Ringwood and Kaufman's model, is to prevail in only one-eighth the volume of the body (i.e. at depths of $\leqslant 0.5 \mathrm{r}$ ), the radius of the body for a density of $3.5 \mathrm{~g} / \mathrm{cm}^{3}$ must be at least $1530 \mathrm{~km}$. For comparison, the moon has a radius of $1738 \mathrm{~km}$. But a body of lunar size, if melted by whatever process, would not cool to $300-400{ }^{\circ} \mathrm{C}$ in the entire age of the solar system, even if heating by $\mathrm{U}$, Th, and $\mathrm{K}^{40}$ is ignored. This fact was first pointed out by Urey (1956) based on calculations by Wood (1963b). Conversely, asteroidal-sized bodies which could cool to the required temperatures, have much too low interior pressure. High pressure and low temperatures simply do not seem to go together. No realistic solution to this dilemma has ever been proposed by advocates of high-pressure hypothesis."

(Anders, E., 1964. Origin, age and composition of meteorites: page 595 (2) Cooling problem)

\subsection{Two Asteroid Belts and Gas Planet Satellites}

In 1802, one of the first people to discover asteroids located in the main asteroid belt, Heinrich Olbers's hypothesized that he was looking at the remains of a destroyed planet. In 1892 Edward Barnard was the first and last person to make a new discovery by directly observing an asteroid captured by Jupiter's gravity, distinctly different than the four Galilean moons. In 1992 Gerard Kuiper was first to observe an object in the outer asteroid belt. At first the outer belt was thought to be the source of periodic comets however, since then studies have shown the outer belt to be stable, leading to the scatter disc and the Oort cloud hypothesis as a source of periodic comet. In the PVE hypothesis, Earth Mark One's violent explosion is identified as the source of periodic comets, the outer belt, the inner belt and the asteroids captured by the gravity of Jovian planets.

\subsection{Particle Size Distinction and Zodiac Lights/Gegenschein}

Duchene et all (2014) reviled the outer belt of Eta Corvi is made of particulates with a minimum grain size of four times the expected blow-out size while the inner belt is made of fine particulates at the blow-out size and smaller during a late stage in development resulting in several possible conclusions. This includes "this difference in dust properties between the two dust belts suggests that the warm (inner) dust cannot be explained by mere transport of grains from the outer belt in, but rather support a rare and more violent recent event.". An asteroid blast pattern of fine particulates within the main belt and larger particulates exceeding blow-out size in the outer belt is consistent with the PVE hypothesis and is exemplified in the Sun's solar system by the existence of Zodiac Lights and a Gegenschein made of fine particulate within the inner solar system on its plateau.

\subsection{Trojan Asteroids}

Trojan asteroids were first observed in 1906 however, they were mathematically hypothesized as far back as 1772 due to the gravity wells created when two sources of gravity orbit one another, 
calculated by and known as Lagrange points (L1-L5). Only Mercury and Saturn currently do not have observed asteroids at their L4 and L5.

In 2005 , the discovery of a single high-inclination $\left(>25^{\circ}\right)$ asteroid in the L4 of Neptune (2005 $\mathrm{TN}_{53}$ ) has led to speculations that Neptune Trojan asteroids could significantly outnumber Jupiter Trojan asteroids by being interpreted as an indication of capturing a thick cloud of asteroids. Though it is likely there are more than the 28 known (as of February 2020) Neptune Trojan asteroids, the active count of Jupiter Trojans are up to 7642 known objects (Minor Planet Center), some up to $40^{\circ}$ off inclination (Pirani, 2019). The PVE Hypothesis is consistent with a greater number of Jupiter Trojans being present and having greater numbers with a higher degree of inclination due to the proximity of Earth Mark One's orbit.

\subsection{Past and Present LHB}

Only two stars are known to have experienced a LHB. Fifty lightyears away, in the Corvis (Crow) constellation, one of those two stars, Eta Corvi is currently experiencing its LHB. This means it is relatively younger- vs the LHB that the Sun of this solar system experienced 4.55 billion year ago. The LHB of this solar system is estimated to have lasted approximately $10-150$ (50\% in the first 3.7) million years before there was a significant decrease in asteroid strikes. The LHB began and abruptly ended 700 million years after the formation of the moons and planets (Gomes, et al., 2005), providing an explanation as to why every object in the Solar System is either heavily cratered or void of craters after early to current lava resurfacing. This leaves no explanation as to why a heavy bombardment would begin so late in the development of the solar system, save the hypothesis that after a long quiescent period the rapid migration of giant planets may have caused it.

Using the Spitzer Space Telescope, Lisse et al. (2012) found the star Eta Corvi (Eta Crv, $\eta$ Corvi, $\eta$ Crv) simultaneously hosts a LHB and multiple asteroid belts. Of significance is that the first extrasolar system observed with a LHB also has a main belt and an outer belt. Four years prior, using images from the Spitzer Space Telescope and the Caltech Submillimeter Observatory, Backman et al. (2008) found the star Epsilon Eridani to have two asteroid belts. However, no mention of an observed LHB suggests further advancements in observation may be required to detect the absence or presence of abundant bombardment craters within the system's past. The activity of a LHB is the least enduring of the observable asteroid blast pattern byproducts most easily seen through a telescope (i.e., main belt with fine particulate, outer belt with larger than blowout particulate, Trojan asteroid groups, a LHB, etc.) orbiting a star hosting a planet that experienced a mass-shedding event.

\subsection{Annual Meteor Showers}

The least understood pattern observed with an attempt to be solved by the PVE Hypothesis is the two orbit changing impacts that occurred after Earth's mass was reduced. Certain patterns expected from such a series of hypothesized impacts do exist. Repeated experiments of a 3D simulations adjusting Earth's perigee and then apogee revealed a consistent recurring pattern 100\% of the time of asteroids (both retrograde and not) returning annually to the locations in their individual new orbits where the asteroid formation event occurred. The debris not ejected from the solar system or impacted into a celestial object would return to the location of their formation once an orbit around the Sun. Thus, the simulated passing of Earth through the first or second orbit changing location created the rhythm of Earth experiencing annual meteor showers. Once both orbit changing maneuvers were completed the simulated Earth Mark Two would experience annual meteor showers during apogee or perigee $100 \%$ of the time depending on the location of the final adjustment becoming the new apogee or remaining the new perigee. Routinely other visual recurring asteroid patterns similar to Halley's comet also developed as well as the loss of wayward moons developing a Ceres-like orbit.

The computer simulation designed to observe hypothetical Earth Mark One following two scripted changes in orbit(3D Solar System Simulator 1.3) reveals patterns within the random orbits of asteroids and dust clouds ejected from three events. After the final adjustment in orbit while at 
perigee with the finial ejection of asteroids, the simulation shows Earth returning once every orbit to the place from where the ejection of asteroids occurred. Although many asteroids and dust clouds were lost due to impact or ejection from the Solar System, the vast majority of asteroids and retrograde asteroids created from this event also return to this place annually in their orbits. Thus, Earth experiences an annual scenario in which it has a greater potential for impact. Even though, after 4.55 billion years, many of the large asteroids have been absorbed into Earth, enough small debris and dust clouds remain that Earth experiences annual interactions in the form of annual meteor showers. The random asteroid simulation also suggests a LHB created in this manor is more likely to impact the Sun and objects in the Inner Solar System (especially Earth's Moon, likely due to the proximity) than other objects in the Solar System.

\subsection{Sunstruck}

Two conflicting hypotheses of the source of the LHB both comply with the Sun being the object most likely struck in the environment of the inner solar system during the LHB, sometimes called the Lunar Cataclysm Period. All the planets in the inner solar system show a history of the LHB, even Earth with its active tectonic movement making it a poor record keeper. Mars's North Pole Basin is a largest impact crater recognized in the solar system however, Earth's Moon has often been a favorite source to use when observing unchanged ancient grounds that reveal the frequency and power of the bombardment that obviously occurred. The hypothesis that giant gas planets performed a late rapid migration, which in turn disturbed otherwise at rest asteroids in the inner asteroid belt (leftover building block hypothesis) would be disturbed enough to cause a bombardment of the inner planets and with them the Sun. The PVE Hypothesis places Earth's Moon directly first in line of all the celestial objects in the solar system to receive the first wave of the LBH when Earth Mark One vaporized and shed the majority of its mass, then the rest of Earth's lost moons. Of the planets, Mars first and then Jupiter would experience that first wave. Against extraordinary odds, the small planet Mars gained two captured satellite asteroids that would last 4.5 billion years. Jupiter's mass and proximity to the thickest and first wave of asteroids in the LHB endowed it with more captured satellite asteroids than any other planet, as well as more confirmed Trojan asteroids. Likely Saturn and all the gas planets gained additional mass to develop their rings from captured LHB asteroids and comets, even though massive rings orbiting exoplanets like J1407b have been observed without asteroid belts and LHBs being present. Every object in the solar system was heavily bombarded, including the largest one, the Sun. The odds are in favor for the Sun to be hit more often in the inner solar system regardless of why the inner asteroid belt bombarded the inner planets 700 million years after they began to form.

\section{Discussing What Can Be Inferred}

A drastic change in Earth's mass/gravity, and forward momentum would be sufficient to release multiple moons of Earth Mark One to become wayward moons, such as Ceres and Pluto, and develop independent orbits around the Sun. Ejection from the Earth-shattering event resulted in the formation of all the asteroids in the LHB and in both asteroid belts. The debris remaining near the event formed the Main Belt and contains smaller than blowout sized particles, as this cloud of small particles spread out in a disc in the inner solar system it became observable to the human eye as the Zodiac Lights and the Gegenschein, also known as the interplanetary dust cloud. The debris ejected farther away (but not far enough to be lost to the Solar System) developed into the Outer Belt with particles four times larger than blowout size. The Trojan asteroid groups developed due to Jupiter's gravitational (Lagrange $4 \& 5$ ) influence on the location of the asteroid formation events. The release of so much of Earth Mark One's mass into countless asteroids also resulted in an extraordinarily small portion of the asteroids falling into stable orbits around the gas planets. Although all the known natural moons whose surfaces are observable are tidally locked, not all known captured asteroids are tidally locked. However, many are, including Phobos and Demos which are the only known examples of stable orbits of asteroids around any terrestrial planet, not including asteroid satellites captured by Earth, observed by Gomes et al. (2005). 


\subsection{Estimate of Earth Mark One's Mass}

Earth Mark One was originally a much larger planet than Earth Mark Two. The total amount of chondritic mass accretion that occurred after the LHB can be measured and used to estimate the mass lost from Earth Mark One during the vaporization event. An analysis of chondrite contamination in Earth's mantle suggests the bombardment contributed $0.3-0.8 \%$ to Earth Mark Two's mass, although Touboul et al. (2015) referred to it as a late accretion to distinguish it from a late veneer. These findings are not altogether inconsistent with the popular explanation (Walker, 2009), suggesting $0.5-0.8 \%$ of the Earth's mass was added after core separation and the giant impact that formed the Moon, based on the abundance of highly siderophile elements (HSE; Brasser et al., 2016). Further analysis of HSE in the lunar mantle indicates the Moon received a much lower accretion of mass (0.02-0.035\%; Brasser et al., 2016; Day and Walker, 2015; Kruijer et al., 2015). Based on W isotopic analysis, the mass added to the Moon from a disproportional late accretion was approximately $0.05 \%$ (Touboul et al., 2015). Martian meteorites indicate chondrite contamination after core formation added an estimated $0.7 \%$ to the planet's mass, which is comparable to the mass added to Earth (Brasser et al., 2016). The average of these three estimates is $0.40 \%$. If every object in the Solar System that existed before the LHB accreted $0.40 \%$ of its mass from chondrite contamination during the LHB, Earth Mark One was $0.40 \%$ of the mass of the Solar System or 0.40\% of 1.0014 solar mass $(\mathrm{M} \odot)$, which is $0.0040 \mathrm{M} \odot$.

However, not every object in the Solar System has been accreted by the LHB equally. The Inner Solar System received a higher percentage of hits early in its development (Brasser et al., 2016). The final estimate assumes the Sun contains most of the mass of the Solar System. Therefore, the Sun will have accreted the $0.40 \%$ that the Inner Solar System received, if not a greater portion. The 3D computer simulation developed during this investigation produced observable random asteroid patterns routinely showing the Sun as being the most likely object to be struck by an asteroid. Thus, one estimated mass of Earth Mark One is the estimated chondritic mass the Sun accreted or $0.0040 \mathrm{M} \odot\left(8.01 \times 10^{27} \mathrm{~kg}\right)$, which is 4.2 times the mass of Jupiter $\left(1.898 \times 10^{27} \mathrm{~kg}\right)$. This estimation does not include the $50 \%$ of chondritic meteorite matrix mass that is not specifically chondrules. Therefore, it can be estimated that the original mass of Earth Mark One is between 4.2 and 8.4 times the mass of Jupiter if not greater. Based on late chondritic accretion and $\mathrm{W}$ isotopic analysis in the Inner Solar System, Earth Mark One was potentially more than $99.98 \%$ larger than present-day Earth (less than $00.02 \%$ of its original mass).

A complete analysis of HSE, chondrite and W isotope ratios requires rock samples from all the terrestrial planets and Jovian moons. Because obtaining rock samples from every object in the Solar System, most notably the Sun, is not possible, a better constrained estimate of the mass accreted into the Sun from the LHB and the proportion of chondrite contamination may allow a more accurate estimation of the mass of Earth Mark One. Confirmation of Earth Mark One's LHB adding to the Sun approximately $0.40 \%$ of its mass may require other methods, such as chemical analysis by spectroscopy.

\section{2/5.3 Distance and Orbital Ratios}

For decades the Titius-Bode Rule has been taboo in the scientific community. However new and increased observations of exo-planets is now challenging the negative stigma of a generalized Titus-Bode effect (Chang, 2010). The PVE Hypothesis provides the Sun's solar system one simple solution to solve the Titius-Bode (Distance Ratio) Rule. What has not been calculated for is the change of Earth's orbit. With Earth calculated at its original orbital position, a generalized distance ratio rule (Titus-Bode Rule) develops very near to correct. An orbital resonance has been observed with the Galilean moons of Jupiter as well as other exoplanets and is not considered as controversial to the scientific community. The Sun's planetary distance ratios and orbital resonances are closer to synergistic when Earth is in its original location as displayed in Table 1. 
Table 1. Planetary Distance Ratios and Orbital Resonances

\begin{tabular}{|l|l|l|l|}
\hline \multicolumn{1}{|c|}{ Planet } & \multicolumn{1}{c|}{ Average Distance in AU } & \multicolumn{1}{c|}{ Distance Ratio } & Orbit Resonance \\
\hline Mercury & 0.4 & 1 & 1 \\
\hline Venus & 0.7 & $1: 1.75$ & $1: 2.5$ \\
\hline Mars & 1.6 & $1: 2.29$ & $1: 3.0$ \\
\hline Ceres (EM1) & 2.8 & $1: 1.75$ & $1: 2.4$ \\
\hline Jupiter & 5.2 & $1: 1.86$ & $1: 2.5$ \\
\hline Saturn & 9.6 & $1: 1.86$ & $1: 2.5$ \\
\hline Uranus & 19.2 & $1: 2.0$ & $1: 2.8$ \\
\hline Neptune & 30.1 & $1: 1.57$ & $1: 1.9$ \\
\hline
\end{tabular}

Averages

Average planetary distance from inner neighboring planet $=1: 1.87$

Mean average planetary distance from inner neighboring planet $=1: 1.75 / 1.86$

Average planetary orbital resonance $=1: 2.5$

Mean average planetary orbital resonance $=1: 2.5$

Mars and Neptune are off of both ratios by the most significant amount and each are missing the influence of a large outside neighbor, Mars by losing Earth Mark One and Neptune by being the outermost planet.

\subsection{Wayward Earth Moons}

The densities of suspected wayward moons can be used to hypothesize their original orbital sequence. As shown in Table 2, the close nature of the densities of Pluto and Charon is consistent with the hypothesis they were neighboring moons of the same planet and moved outward at the same time during the same event, establishing a new binary relationship with each other. Earth and the Moon sharing a binary orbit is also consistent with the use of density to hypothesize the original lunar sequence due to the Moon being the most dense of Earth's moons, therefore the closest moon to Earth. When Earth's mass was reduced, it lost gravitational dominance over all except the closest moon. 
Table 2. Densities of Suspect Wayward Moons

\begin{tabular}{|l|l|l|}
\hline \multicolumn{1}{|c|}{ Moon/Wayward Moon } & \multicolumn{1}{|c|}{ Density } & \multicolumn{1}{c|}{ References } \\
\hline Moon & $3.34 \mathrm{~g} / \mathrm{cm}^{3}$ & Wieczorek, 2006 \\
\hline Eris & $2.3 \pm 0.3 \mathrm{~g} / \mathrm{cm}^{3}$ & Brown \& Schaller, 2007 \\
\hline Quaoar & $1.99 \pm 0.46 \mathrm{~g} / \mathrm{cm}^{3}$ & Braga-Ribas, 2013 \\
& $2.18+0.43-0.36 \mathrm{~g} / \mathrm{cm}^{3}$ & Fornasier et al., 2013 \\
\hline Ceres & $2.161 \pm 0.009 \mathrm{~g} / \mathrm{cm}^{3}$ & Lakdawalla, 2015 \\
\hline Pluto & $1.88 \mathrm{~g} / \mathrm{cm}^{3}$ & Stern et al., 2017 \\
\hline 2007 OR10 & $1.72 \pm 0.16 \mathrm{~g} / \mathrm{cm}^{3}$ & Kiss et al., 2018 \\
\hline Charon & $1.71 \mathrm{~g} / \mathrm{cm}^{3}$ & Stern et al., 2017 \\
\hline 90482 Orcus & $1.53+0.15-0.13 \mathrm{~g} / \mathrm{cm}^{3}$ & Rambaux et al., 2017 \\
\hline Makemake* & $1.4-3.2 \mathrm{~g} / \mathrm{cm}^{3}<3.05$ & Brown, 2013b \\
\hline 2004 SB60 & $\mathrm{g} / \mathrm{cm}^{3}$ & \\
\hline 20000 Varuna & $1.29+0.29-0.23 \mathrm{~g} / \mathrm{cm}^{3}$ & Rambaux et al., 2017 \\
\hline 2002 UX25 & $0.992 \mathrm{~g} / \mathrm{cm}^{3}$ & Lacerda \& Jewitt, 2016 \\
\hline Sedna* & $0.82 \pm 0.11 \mathrm{~g} / \mathrm{cm}^{3}$ & Brown, 2013a \\
\hline 2002 AW197* & $*$ & Rambaux et al., 2017 \\
\hline 2002 TC302* & $*$ & $*$ \\
\hline 1996 TO66* & $*$ & $*$ \\
\hline 1996 TL66* & $*$ & $*$ \\
\hline 19521 Chaos* & $*$ & $*$ \\
\hline * More data needed. & $*$ & $*$ \\
\hline & $*$ & $*$ \\
\hline
\end{tabular}

\section{$5.5 \mathrm{CHON}$}

Life as we currently understand it is carbon based, possibly due to carbons uncommon ability to make 4 separate atomic bonds however, there are other atomic biomarkers that are associated with our understanding of life (Airapetian et al., 2017). oxygen $\left(\mathrm{O}_{2}\right)$, ozone $\left(\mathrm{O}_{3}\right)$, water vapor $\left(\mathrm{H}_{2} \mathrm{O}\right)$, carbon dioxide $\left(\mathrm{CO}_{2}\right)$, nitrous oxide $\left(\mathrm{N}_{2} \mathrm{O}\right)$, and methane $\left(\mathrm{CH}_{4}\right)$.

To identify Earth-like planets for the purposes of supporting habitats, ecosystems and life as we know it, researchers can use the defining characteristics of (1) magnetic shielding, (2) warmth, and (3) food resulting from a supercharged geothermal core in addition to the favored characteristics of (4) similar gravity, an (5) appropriate distance from a star, and (6) the presence of water in all three phases. Further investigation of the stars Eta Corvi and Epsilon Eridani (as well as every other star with two asteroid belts) are required to test if these 6 observed phenomena are a reliable method for locating life-inhabiting exo-planets. Although space and ground-based telescopes already exist to allow observation of Eta Corvi, Epsilon Eridani and any other star located with two distinct asteroid belts, even more powerful telescopes are in development that would allow the spectral observation of C.H.O.N. on an orbiting planet (possibly with the upcoming James Webb Space Telescope).

\section{Conclusion}

A 0.86-Billion-degree Kelvin explosion strikes Earth Mark One (with a mass of at least 4.2 Jupiter's), reduced its mass by at least $99.98 \%$ and homogenizes on a planet-scale ${ }^{182} \mathrm{~W} \varepsilon$ isotopes. At 100+ times the density of a nebular cloud the Late Heavy Bombardment suddenly freezes and begins to heavily bombard the inner solar system. Many asteroids with less mass stay near the asteroid formation event to develop the inner asteroid belt. Many asteroids with greater mass traveled out to become the Trojan Groups, the satellites of gas planets and the outer asteroid belt. The moons of Earth free from Earth's gravity travel across the solar system in various directions, some staying together in a new binary orbit while outgassing identical vapor. Within a 5 million year window, a 
1,200-1,900 ${ }^{\circ} \mathrm{C}$ second and third asteroid formation event results in the orbit changing course corrections needed to change Earth Mark Two's orbit away from the developing asteroid belt, to between Mars and Venus, setting up annual meteor showers for Earth.

The physical evidence supports the PVE hypothesis is viable as an explanation of the origins of Earth's super charged geothermal core, a manor to reclassify many objects in the solar system and as a way of finding planets with a similar supercharged geothermal core capable of supporting ecosystems for billions of years.

Understanding the history of Earth's formation and reformation event is important for understanding why the Earth has a supercharged geothermal core. However, the ability to use the knowledge afforded by the PVE Hypothesis to seek out Earth-like worlds is of greater potential in terms of the future of space travel for humans and all life-forms inseparable from the human experience.

\section{Acknowledgments}

Thanks are owed to retired Principal Librarian Eloise R. Hurst, clerical editor, former editor and chief Florida Journal of Public Health Clara Lawhead MS,RD,LD,FADA, editor, and Timothy Ruger, APA editor. A huge thank you goes to Lindsay Hall, 3D simulation developer. A humble thank you goes to scientific editor Dr. Benjamin Turner, scientific editor Mr. Vic Ridgley, and scientific editor Dr. Arpad Lorberer. Thanks also to Dr. Antonio Paris and the staff at edit911.com. The last thank you goes to Antoine Bonnet for digitally creating the figure used in this article.

\section{References}

[1] Alexander, C. M. O'D., Grossman, J. N., Ebel, D. S. et al., 2008. The formation conditions of chondrules and chondrites. Science 320 (5883) 1617-1619. doi:10.1126/science.1156561.

[2] Alley, C. O., Bender, P. L, Chang, R. F. et al., 1969. Laser ranging retroreflector. In: Apollo 11: Preliminary Science Report, NASA SP-214. NASA, Washington, DC, pp. 163-182.

[3] Amelin, Y., Krot, A. N., Hutcheon, I. D. et al., 2002. Lead isotopic ages of chondrules and calcium-aluminum-rich inclusions. Science $297 \quad$ (5587) 1678-168. doi:10.1126/science.1073950.

[4] Amelin, Y., Krot, A., 2007. Pb isotopic age of the Allende chondrules. Meteoritics \& Planetary Science 42 (7-8) 1321-1335. doi:10.1111/j.1945-5100.2007.tb00577.x.

[5] Anders, E., 1964. Origin, age and composition of meteorites. Space Science Reviews 3 (5-6) 583-714. doi:10.1007/BF00177954.

[6] Avice, G., Meier, M. M. M., Marty, B. et al., 2015. A comprehensive study of noble gases and nitrogen in Hypatia, a diamond-rich pebble from SW Egypt. Earth and Planetary Science FFLetters 432C (October) 243-253. doi:10.1016/j.epsl.2015.10.013.

[7] Airapetian, V.S., Jackman, C.H., Mlynczak, M., 2017. Atmospheric Beacons of Life from Exoplanets Around G and K Stars. Sci Rep 7, 14141. https://doi.org/10.1038/s41598-01714192-4 arXiv:astro-ph/0004117v1 (https://arxiv.org/abs/astro-ph/0004117)

[8] Backman, D., Marengo, M., Stapelfeldt, K. et al., 2008. Epsilon Eridani's planetary debris disk: Structure and dynamics based on Spitzer and CSO observations. The Astrophysical Journal 690 (2) 1522-1538. doi:10.1088/0004-637X/690/2/1522

[9] Belyanin, G. A., Kramers, J. D., Andreoli, M. A. G. et al., 2017. Petrography of the carbonaceous, diamond-bearing stone "Hypatia" from southwest Egypt: A contribution to the debate on its origin. Geochimica et Cosmochimica Acta 223 (February 15) 462- 492. doi:10.1016/j.gca.2017.12.020. 
[10] Bouvier, A., Wadhwa, B., 2010. The age of the solar system redefined by the oldest $\mathrm{Pb}-\mathrm{Pb}$ age of a meteoritic inclusion. Nature Geoscience 3 637-641. doi:10.1038/ngeo941.

[11] Braga-Ribas,F., Sicardy, B., Ortiz, J., et al., 2013. The size, shape, albedo, density, and atmospheric limit of transneptunian object (50000) Quaoar from multi-chord stellar occultations. The Astrophysical Journal 773 (1) 26. doi: 10.1088/0004-637X/773/1/26

[12] Brasser, R., Mojzsis, S. J., Werner, S. C. et al., 2016. Late veneer and late accretion to the terrestrial planets. Earth and Planetary Science Letters 455 (December 1) 85-93. doi.org/10.1016/j.epsl.2016.09.013.

[13] Brown, M. E., 2013a. The density of mid-sized Kuiper belt object 2002 UX25 and the formation of the dwarf planets. Astrophysics arXiv:1311.0553 [astro-ph.EP]. doi:10.1088/2041- 8205/778/2/L34

[14] Brown, M. E., 2013b. On the size, shape, and density of dwarf planet Makemake. Retrieved from https://arxiv.org/pdf/1304.1041v1.pdf

[15] Brown, M. E., Schaller, E. L., 2007. The mass of dwarf planet Eris. Science 316, 1585.

[16] Canup, R., Asphaug, E., 2001. Origin of the Moon in a giant impact near the end of the Earth's formation. Nature 412 (6848) 708-712. doi:10.1038/35089010.

[17] Clague, D. A., Dalrymple, G. B., 1987. The Hawaiian-Emperor volcanic chain. Part 1. Geologic evolution. In R. W. Decker, T. L. Wright, P. H. Stauffer (Eds.), Volcanism in Hawaii: Papers to Commemorate the 75th Anniversary of the Founding of the Hawaiian Volcano Observatory. United States Geological Survey Professional Paper 1350, pp. 5-100.

[18] Chang, Heon-Young. (2010). Titius-Bode's Relation and Distribution of Exoplanets. Journal of Astronomy and Space Sciences. 27. 1-10. 10.5140/JASS.2010.27.1.001

[19] Connelly, J. N., Bizzarro, M., Krot, A. N. et al., 2012. The absolute chronology and thermal processing of solids in the solar protoplanetary disk. Science 338 (6107) 651-655. doi:10.1126/science. 1226919.

[20] Currie, D. G., Dell'Agnello, S., Delle Monache, G. O. et al., 2013. A lunar laser ranging retroreflector array for the 21st century. Nuclear Physics B Proceedings Supplements 243244 (October-November) 218-228. doi:10.1016/j.nuclphysbps.2013.09.007.

[21] Davies, J. H., Davies, D. R., 2010. Earth's surface heat flux. Solid Earth 1 (1) 5-24. doi:org/10.5194/se-1-5-2010.

[22] Day, J. M. D., R. J. Walker, 2015. Highly siderophile element depletion in the Moon. Earth and Planetary Science Letters 423, 114-124. doi:10.1016/j.eps1.2015.05.001.

[23] Domeier, M., Torsvik, T. H., 2014. Plate tectonics in the late Paleozoic. Geoscience Frontiers 5 (3) 303-350. doi:org/10.1016/j.gsf.2014.01.002.

[24] Dones, L., Weissman, P. R., Levison, H. F. et al., 2004. Oort Cloud formation and dynamics. In:

[25] D. Johnstone, F. C. Adams, D. N. C. Lin et al. (Eds.), Star Formation in the Interstellar Medium: In Honor of David Hollenbach, Chris McKee and Frank Shu, ASP Conference Proceedings, vol. 323. Astronomical Society of the Pacific, San Francisco, 371.

[26] G. Duchene, P. Arriaga, M. Wyatt, G. Kennedy, B. Sibthorpe, C. Lisse, W. Holland, J. Wisniewski, M. Clampin, P. Kalas, C. Pinte, D. Wilner, M. Booth, J. Horner, B. Matthews, J. Greaves. 2014. Spatially resolved imaging of the two-component eta Crv debris disk with Herschel. The Astrophysical Journal 784 (2).Dye, S. T., 2012. Geoneutrinos and the radioactive power of the Earth. Reviews of Geophysics 50 (3) -19 . doi:10.1029/2012RG000400. 
[27] Fernandez, J. A., 1980. On the existence of a comet belt beyond Neptune. Monthly Notices of the Royal Astronomical Society 192 (3) 481-491. doi:10.1093/mnras/192.3.481

[28] Fornasier, S., Lellouch, E., Müller, T., et al., 2013. TNOs are cool: A survey of the transNeptunian region. VIII. Combined Herschel PACS and SPIRE observations of 9 bright targets at 70--500 micron. Astronomy and Astrophysics 555 A15 arXiv:1305.0449 [astro- ph.EP]. doi: 10.1051/0004-6361/201321329

[29] Gando, A., Gando, Y., Ichimura, K. et al. Partial radiogenic heat model for Earth revealed by geoneutrino measurements. Nature Geosci 4, 647-651 (2011). https://doi.org/10.1038/ngeo1205

[30] Gomes, R., Levison, H. F., Tsiganis, K. et al., 2005. Origin of the cataclysmic Late Heavy Bombardment period of the terrestrial planets. Nature 435 (May) 466-469. doi:10.1038/nature03676.

[31] Grady, M. M., 2000. Catalogue of meteorites 5th ed. Cambridge, UK: Cambridge University Press.

[32] Halliday, A. N., 2000. Hf-W chronometry and inner solar system accretion rates. Space Science Reviews 92 (1) 355-370. doi:10.1023/A:1005280220751.

[33] Herschel, W., 1807. Observations on the nature of the new celestial body discovered by Dr.

[34] Olbers, and of the comet which was expected to appear last January in its return from the Sun. The Academy, London, pp. 260-266.

[35] Hess, W. N., 1968. The radiation belt and magnetosphere. Blaisdell, Waltham, MA. Hewins, R. H,. Radomsky, P. M. 1990. Temperature conditions for chondrule formation.

[36] Meteoritics 25 (4) 309-318. doi:10.1111/j.1945-5100.1990.tb00715.x.

[37] Ignasi, R., 2010. The Sun and stars as the primary energy input in planetary atmospheres. In: Solar and Stellar Variability: Impact on Earth and Planets, Proceedings of the International Astronomical Union 5 (264) 3-18. doi:10.1017/S1743921309992298.

[38] Isherwood, R. J., Jozwiak, L. M., Jansen, J. C. et al., 2012. The volcanic history of Olympus Mons from paleo-topography and flexural modeling. Department of Geophysics and Center for Space Resources, Colorado School of Mines, Golden, CO.

[39] Karner, G. D., Watts, A. B., 1983. Gravity anomalies and flexure of the lithosphere at mountain ranges. Journal of Geophysical Research 88 (B12) 10449-10477. doi:10.1029/JB088iB12p10449.

[40] Keiko, N., Messenger, S., Keller, L. P. et al., 2006. Organic globules in the Tagish Lake meteorite: Remnants of the proto-solar disk. Science 314 (5804) 1439-1442. doi:10.1126/science. 1132175

[41] Kirkwood, D., 1867. Meteoric astronomy: A treatise on shooting-stars, fireballs, and aerolites. J.B. Lippincott, Philadelphia.

[42] Kiss, C., Marton, G., Parker, A. et al., 2018. The mass and density of the dwarf planet 2007 OR10. 50th annual meeting of the AAS Division of Planetary Sciences. abstract 311.02. Retrieved 21 September 2018. Retrieved from https://aas.org/meetings/dps50

[43] Krot, A. N, Amelin, Y., Bland, P. et al., 2009. Origin and chronology of chondritic components: A review. Geochimica et Cosmochimica Acta 73 (17) 4963-4997. doi:10.1016/j.gca.2008.09.039.

[44] Kruijer, T. S., Kleine, T., Fischer-Gödde, M. et al., 2015. Lunar tungsten isotopic evidence for the late veneer. Nature 520 (April 23) 534-537. doi:10.1038/nature14360. 
[45] Lacerda, P., Jewitt, D., 2016. Densities of solar system objects from their rotational lightcurves. Astrophysics arXiv:astro-ph/0612237. doi:10.1086/511772.33544r f

[46] Lakdawalla, E., 2015, November 12. DPA 2015: First reconnaissance of Ceres by Dawn [blog post]. The Planetary Society. Retrieved from http://www.planetary.org/blogs/emilylakdawalla/2015/dps15-1112-ceres.html

[47] Lisse, C. M., Wyatt, M. C., Chen. C. H. et al., 2012. Spitzer evidence for a late-heavy bombardment and the formation of ureilites in $\eta$ Corvi at $\sim 1$ gry. The Astrophysical Journal 747 (93) 25. doi:10.1088/0004-637X/747/2/93.

[48] MacKenzie, D., 2003. The big splat or how our moon came to be. John Wiley \& Sons, Hoboken, NJ.

[49] MacPherson, G. J., Simon, S. B., Davis, A. M. et al., 2005. Calcium-aluminum-rich inclusions: Major unanswered questions. In: A. N. Krot, E. R. D. Scott, and B. Reipurth (Eds.), Chondrites and the Protoplanetary Disk, Astronomical Society of the Pacific Conference Series, vol. 341. Astronomical Society of the Pacific, San Francisco, 225-250.

[50] Magill, F., 1990. Magill's survey of science: Earth science series. Vol. 2. Salem Press, Hackensack, NJ.b

[51] Minorplanetcenter.net/iau/lists/Trojans.html

[52] National Research Council, 2011. The primitive bodies: Building blocks of the solar system. In: cVision and voyages for planetary science in the decade 2013-2022. The National Academies Press, Washington, DC 87-110. doi.org/10.17226/13117.

[53] O'Leary, M., 2008. Anaxagoras and the origin of panspermia theory. iUniverse , Bloomington, IN.

[54] Ott, U., 2002. Isotopes of colatiles in pre-solar grains. Space Science Reviews 106 (1-4) 3348, doi:10.1023/A:1024621200898.

[55] Pirani, S., Johansen,A. and Mustill, A. J., 2019. On the Inclinations of the Jupiter Trojans.

[56] Astronomy \& Astrophysics 631 (2019): A89. Crossref. Web.

[57] Potemine,I. Y., 2010. Transit of Luyten 726-8 within 1 ly from Epsilon Eridani. Eprint arXiv:1004.1557

[58] Quitté, G., Halliday, A. N., Meyer, B. S. et al., 2007. Correlated iron 60, nickel 62, and zirconium 96 in refractory inclusions and the origin of the solar system. The Astrophysical Journal 655 (1) 678-684, doi:10.1086/509771.

[59] Rambaux, N., Baguet, D., Chambat, F. et al., 2017. Equilibrium shapes of sarge transNeptunian objects. The Astrophysical journal letters, Bristol : IOP Publishing, 850 (1), L9.

[60] Rayman, M., 2015. Now appearing at a dwarf planet near you: NASA's Dawn mission to the Asteroid Belt. Silicon Valley Astronomy Lectures. Foothill College, Los Altos, CA.

[61] Sanctis, M. C. De, Ammannito, E., Raponi, A. et al., 2015. Ammoniated phyllosilicates with a likely outer solar system origin on (1) Ceres. Nature 528 (December 10) 241-244. doi:10.1038/nature16172

[62] Schmidt, L. J., 2004. Sensing remote volcanoes. National Aeronautics and Space Administration. https://earthdata.nasa.gov/user-resources/sensing-our-planet/sensingremote-volcanoes.

[63] Schrader, D. L., Fu, R. R., Desch S. J., 2016. Evaluating chondrule formation models and the protoplanetary disk background temperature with low-temperature, sub-silicate solidus chondrule cooling rates, LPI Contribution No. 1903. 47th Lunar and Planetary Science Conference, March 21-25, 2016, The Woodlands, TX, p. 1180. 
[64] Stern, S. A., Grundy, W., McKinnon, W. B., et al., 2017. The Pluto system after New Horizons. Annual Reviews of Astronomy ans Astrophysics 2018 arXiv:1712.05669 [astroph.EP]. doi:10.1146/annurev-astro-081817-051935

[65] Touboul, M., Puchtel, I. S., Walker, R. J., 2015. Tungsten isotopic evidence for disproportionate late accretion to the Earth and Moon. Nature 520 (April) 530-533, doi:10.1038/nature14355.

[66] Von Humboldt, A., 1850. Cosmos: A sketch of a physical description of the universe. 1. Harper \& Brothers, New York, 44.

[67] Walker, R. J., 2009. Highly siderophile elements in the Earth, Moon and Mars: Update and implications for planetary accretion and differentiation. Chemie der Erde 69, 101-125.

[68] Wegener, A., 1912. Die Herausbildung der Grossformen der Erdrinde (Kontinente und Ozeane), auf geophysikalischer Grundlage. In Petermanns Geographische Mitteilungen 50 (1) 185-195, 253-256, 305-309.

[69] Weijermars, R., 1989. Global tectonics since the breakup of Pangea 180 million years ago: Evolution maps and lithospheric budget. Earth Science Reviews 26 (1-3) 113-162. doi:10.1016/0012-8252(89)90020-2

[70] Weizsäcker, C. F., 1948. The rotation of cosmic gas masses. Zeitschrift für Naturforschung A 3 (8-11) 524-539. doi:10.1515/zna-1948-8-1118.

[71] Wieczorek,M.A., 2006. The constitution and structure of the lunar interior. Reviews in Mineralogy and Geochemistry 60 (1) 221-364. doi:10.2138/rmg.2006.60.3

[72] Williams, D. A., Keszthelyi, L. P., Crown, D. A. et al., 2011. Geologic map of Io: U.S. Geological Survey Scientific Investigations Map 3168, 25. Available at https://pubs.usgs.gov/sim/3168/.

[73] Windley, B. F., 1970. Anorthosites in the early crust of the Earth and on the Moon. Nature 226 (5243) 333-335. doi:10.1038/226333b0.

[74] Wood, J. A., 1963a. Nature 194, 127.

[75] Wood, J. A., 1963b, unpublished work.

[76] Yoneda, S., Grossman, L., 1995. Condensation of CaO-MgO-AI203-SiO2 liquids from cosmic gases. Geochimica et Cosmochimica Acta 59 (16) 3413-3444.

[77] Youngl, E. D., Warren, P. H., Rubie, D. C. et al., 2016. Oxygen isotopic evidence for vigorous mixing during the Moon-forming giant impact. Science 351 (6272) 493-496. doi:10.1126/science.aad0525. 